\title{
Electronic Structure Calculations Using An Adaptive Wavelet Basis
}

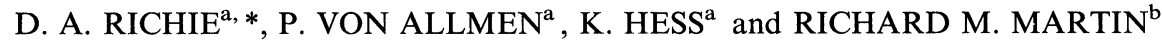 \\ ${ }^{a}$ Beckman Institute for Advanced Science and Technology, University of Illinois, Urbana, Illinois 61801; \\ ${ }^{\mathrm{b}}$ Department of Physics, University of Illinois, Urbana, Illinois 61801
}

\begin{abstract}
The use of a wavelet basis can lead to efficient methods for performing $a b$ initio electronic structure calculations of inherently localized structures. In this work wavelets are used to construct an adaptive basis which is optimized dynamically throughout the calculation. The computational effort of such a method should scale linearly with the number of basis functions. The adaptive basis is tested for the case of bulk Si using only a local s-pseudopotential.
\end{abstract}

Keywords: Wavelets, multiresolution analysis, electronic structure, density functional theory, adaptive basis

\section{INTRODUCTION}

Much progress has been made in the area of $a b$ initio electronic structure calculations using the local density approximation (LDA) of density functional theory (DFT). Within DFT the total energy is considered to be a functional of the electron density. The electronic ground state is found by minimizing this total energy, leading to the solution of the Kohn-Sham single-particle Schrödinger equation.

Despite its advantages, the commonly used plane wave (PW) basis possesses serious drawbacks when applied to localized systems. Examples of such systems include those with deep atomic pseudopotentials, e.g., oxygen, or structures of a localized nature, e.g., molecules, surfaces and interfaces. In such cases a prohibitively high energy cutoff is required to provide the necessary resolution. Due to the delocalized nature of the PW basis functions, this increase in resolution is applied globally even though the increased resolution is needed in an essentially localized region of space.

As a result of these problems various alternatives to the PW method have been proposed. Most can be classified as real-space methods. These include the use of adaptive curvilinear coordinates [1], finite difference [2] and multigrid [3] methods as well as wavelet bases. It is the use of a wavelet basis which will be the topic of this paper.

${ }^{*}$ Corresponding author. 
The use of a wavelet basis for electronic structure calculations was first proposed by Cho et al. [4] where non-orthonormal wavelets were used. The work of Wei and Chou [5] used the compact orthonormal wavelets constructed by Daubechies [6]. The same Daubechies wavelets are used in our work. In addition, both Ref. [4] and Ref. [5] used a basis that was predetermined by simple physical arguments and remained fixed throughout the calculation. In our work a truly adaptive basis is used which does not rely on any $a$ priori assumptions, but instead allows the calculation to dynamically determine the optimum basis for each electronic orbital. Our work is also unique in that the entire calculation is performed in a wavelet basis. This creates the possibility for a general method where both the execution time and storage scale linearly with the number of basis functions used to represent each electronic orbital.

\section{WAVELET BASIS}

A detailed discussion of wavelets can be found in Ref. [6]. A wavelet basis forms a multiresolution analysis (MRA). Such an analysis represents a function as the limit of increasingly finer approximations, with each approximation being the result of smoothing, or averaging, the function over some fixed length scale. The result is a series of embedded function spaces $V_{m}$ such that

$$
\ldots V_{m+2} \subset V_{m+1} \subset V_{m} \subset V_{m-1} \subset V_{m-2} \ldots
$$

A basis spanning each function space, or approximation space, can be constructed from the translations of some localized function $\phi(x)$ known as the scaling function. Thus, a basis for the approximation space $V_{m}$ can be written $\left\{\phi_{m n}(x)=2^{m / 2} \phi\left(2^{m} x-n\right), n \in Z\right\}$. The corresponding characteristic length scale is $\sim 2^{-m}$. The MRA can be seen to be generated by this 'mother scaling function' $\phi(x)$. The choice of the mother scaling function is in fact the beginning of the MRA which generates a wavelet basis.
Next, consider the projection of a function onto two successive approximation spaces $V_{m}$ and $V_{m+1}$. Information is lost when going from the finer approximation to the coarser one. This leads to the introduction of an additional function space $W_{m}$ defined to be the orthogonal complement of $V_{m}$ in $V_{m+1}$, i.e., $V_{m} \oplus W_{m}=V_{m+1}$ and $V_{m} \perp W_{m}$. This function space, or detail space, $W_{m}$ contains the information which is lost when making the coarser approximation. Conversely, the finer approximation of a function can be constructed from its projection onto the coarser approximation space plus its projection onto the associated detail space.

A basis spanning each detail space can be constructed from the translations of some localized function $\psi(x)$, known as a wavelet. Thus, $W_{m}$ may be spanned by $\left\{\psi_{m n}=2^{m / 2} \psi\left(2^{m} x-n\right)\right.$, $n \in \boldsymbol{Z}$ \}. Here the function $\psi(x)$ is known as the 'mother wavelet' and is directly related to the associated mother scaling function used to generate the MRA. It is the combination of scaling functions and wavelets which are commonly referred to as a wavelet basis.

There exist a wide variety of wavelets constructed to have different properties. In this work we use two types of wavelets, specifically the wavelet known as Daubechies-6 $\left(\mathcal{D}_{6}\right)$ and the Haar wavelet. In Figure 1 and Figure 2 the mother scaling function and mother wavelet are shown for $\mathcal{D}_{6}$. Both wavelets are compact, complete and orthonormal. In addition, the $\mathcal{D}_{6}$ wavelet has a continuous first and second derivative.

The expansion of some function $f(x)$ onto a wavelet basis will therefore have the form of an expansion onto the translations of some coarse scaling function plus the detail of the expansion onto the translations and dilations of the associated wavelet,

$$
f(x)=\sum_{n=-\infty}^{+\infty} \bar{f}_{0 n} \phi_{0 n}(x)+\sum_{m=0}^{+\infty} \sum_{n=-\infty}^{+\infty} f_{m n} \psi_{m n}(x) .
$$

The infinite sums over all translations can be removed by imposing periodic boundary conditions, which leads to periodized wavelets. A three 


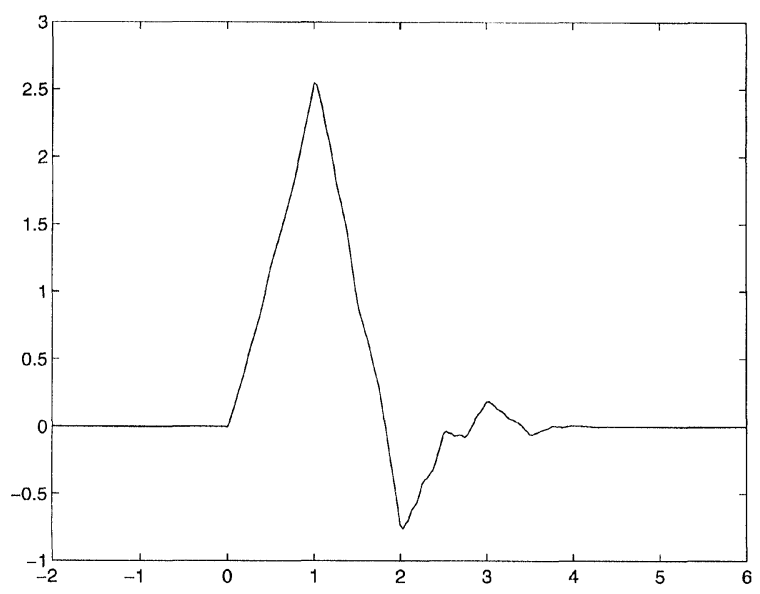

FIGURE 1 Real space plot of the Daubechies-6 mother scaling function.

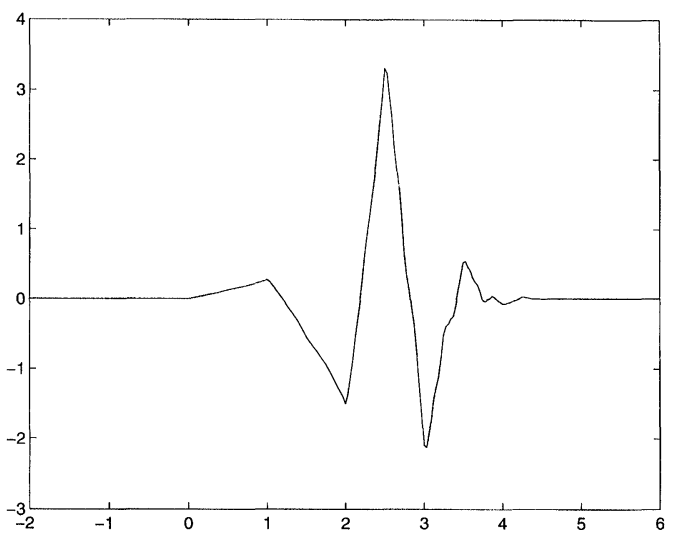

FIGURE 2 Real space plot of the Daubechies-6 mother wavelet.

dimensional basis can be constructed from tensor products of the one dimensional basis.

One of the key properties of a wavelet basis is its ability to represent the localized detail of a function using a small number of wavelets. This is a result of the fact that the wavelet expansion coefficients, $f_{m n}$ in Eq. (1), will decay rapidly with increasing $m>M$, where the local smoothness of the function can be characterized by the length scale $\sim 2^{-M}$. If the magnitude of the expansion coefficient is less than some threshold which is small compared to the norm of the function, then no further detail is needed in that region. This leads to the idea of a 'compressed basis', the structure of which is determined by the above criteria, forming a minimal basis set representation for a specific function. Such a compressed basis will be used to represent the electronic orbitals in the electronic structure calculation.

\section{CALCULATION}

The electronic structure of the system is determined here using the local density functional theory neglecting the spin. Therefore we determine the electronic ground state by solving the KohnSham equation ${ }^{1}$,

$$
H_{\mathrm{KS}} \psi_{i}(r)=E_{i} \psi_{i}(r)
$$

where the electron density is given by $\rho(r)$ $=2 \sum_{i=1}^{N}\left|\psi_{i}(r)\right|^{2}$ for a $2 \mathrm{~N}$-electron system since each state $\psi_{i}(r)$ is doubly-occupied [7]. Details of this equation and methods of solution can be found in Ref. [8].

A wavelet based Laplacian operator is needed for the kinetic energy term of the Kohn-Sham Hamiltonian as well as in the solution of Poisson's equation. This Laplacian operator is performed by matrix multiplication. The elements of the differentiation matrix are pre-calculated using the method described in Ref. [9] The solution of Poisson's equation in a wavelet basis is by itself an interesting topic. The efficiency of the wavelet basis in this problem is well established [10].

The operation involving the multiplication of the wave function by the local potential is performed using the following algorithm. First, both the wave function and the local potential are mapped from a $\mathcal{D}_{6}$ wavelet representation to a

\footnotetext{
${ }^{1}$ Atomic units are used throughout.
} 
Haar wavelet representation using a transform. Then, the two functions are multiplied in their Haar representations. The product is then mapped back to a $\mathcal{D}_{6}$ wavelet representation using an inverse to the transform mentioned above. Thus, the multiplication is performed entirely in a wavelet basis.

The electronic ground state of the system is found by solving Eq. (2) iteratively. The wavelet basis is held fixed until convergence is achieved. The basis is then compressed, removing unnecessary basis functions. Then basis functions are added to effectively double the resolution globally. This is necessary to allow the basis to 'grow' to the optimum structure. With this new basis, the total energy is again minimized and the process is repeated.

\section{RESULTS}

We have tested this method by applying it to a supercell containing $8 \mathrm{Si}$ atoms located at their bulk positions and using a local s-pseudopotential. First, a simpler calculation is performed where the multiplication of the electronic orbital with the local potential is performed by first mapping both functions to real space via a fast wavelet transform, multiplying the two functions and then mapping back to wavelet space. This procedure is similiar to that used in Ref. [5]. In this calculation there is no compression since the full cubic grid must be used. This "full grid" method is performed for increasing grid sizes of $4^{3}, 8^{3}, 16^{3}$ and $32^{3}$. The results are shown in Figure 3. The correct degeneracies (1-66-3) of the 16 eigenvalues are achieved consistent with the symmetry of the problem.

Next, the same calculation is performed with an adaptive wavelet basis. Here, the multiplication is performed using the algorithm, described above, where the entire calculation is performed in a wavelet basis. The correct degeneracies are again achieved to within $10^{-4}$ a.u. The adaptive basis converges to the correct eigenvalues. For the three lowest levels the convergence requires less basis functions.

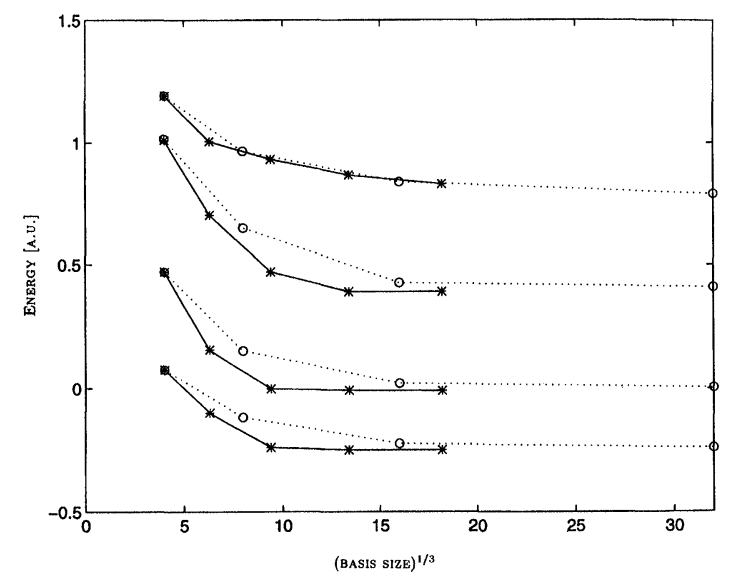

FIGURE 3 Eigenvalues of the Kohn-Sham Hamiltonian for a supercell containing $8 \mathrm{Si}$ atoms. A local s-pseudopotential is used. The eigenvalues are plotted vs. (basis size) ${ }^{1 / 3}$. Comparision is between the adaptive and non-adaptive calculations indicated by the solid and dashed lines, respectively.

\section{CONCLUSION}

We have demonstrated that it is possible to minimize the total energy in an electronic structure calculation while simultaneously optimizing the wavelet basis set used to represent each electronic orbital. This general method should lead to efficient, potentially linearly scaling electronic structure calculations. Furthermore, if the atoms are allowed to move as in a molecular dynamic simulation, the adaptive basis should adjust to the needs of the structure. Presently, the various corrections resulting from exchange-correlation and non-local effects need to be implemented to perform calculations precise enough to compare with other more established methods. The advantages of an adaptive wavelet basis should lead to efficient methods when applied to larger, localized structures.

\section{Acknowledgements}

This work was supported by the Office of Naval Research and the Army Research Office. 


\section{References}

[1] Gygi, F. and Galli, G. (1995). Phys. Rev., B52, R2229.

[2] Chelikowsky, J. R., Troullier, N. and Saad, Y. (1994). Phys. Rev., B50, 11355.

[3] Briggs, E. L., Sullivan, D. J. and Bernholc, J. (1996). Phys. Rev., B54, 14362.

[4] Cho, K., Arias, T. A., Joannopoulos, J. D. and Lam, Pui K. (1993). Phys. Rev. Lett., 71, 1808.

[5] Wei, S. and Chou, M. Y. (1996). Phys. Rev. Lett., 76, 2650.

[6] Daubechies, I. (1992). Ten Lectures on Wavelets (SIAM, Philadelphia).

[7] Kohn, W. and Sham, L. J. (1965). Phys. Rev., A140, 1133.

[8] Stich, I., Car, R., Parrinello, M. and Baroni, S. (1989). Phys. Rev., B39, 4997.

[9] Beylkin, G. (1992). SIAM J. Numer. Anal., 6, 1716.

[10] Goedecker, S. and Ianov, O. V., unpublished.

\section{Author's Biographies}

David A. Richie received his B.S. degree in electrical engineering from the University of Wisconsin-Madison. He is currently a doctorate student at the University of Illinois at UrbanaChampaign where he is working towards a Ph.D. in physics. He was previously a student fellow at Sandia National Laboratories where his research involved the study of vertical cavity surface emitting lasers. $\mathrm{He}$ is currently studying the use of adaptive wavelet bases in electronic structure calculations and molecular dynamics simulations.

Paul von Allmen obtained his B.S. and Ph.D. in Physics from the Swiss Federal Institute of Technology at Lausanne, Switzerland. He joined the Zurich IBM Research Laboratory as a Postdoctoral Research Associate in 1990 and was an Invited Scholar at the Beckman Institute, University of Illinois at Urbana-Champaign from 1992 to 1997 . He presently is a Senior Scientist at the Motorola Phoenix Corporate Research La- boratories. His interests include subband structures and many-body effects in confined electron systems and dynamical properties of nanostructures and surfaces. He has performed ab initio simulations of hydrogen and deuterium desorption from a silicon surface. He presently investigates the field emission properties of carbon materials.

Karl Hess has dedicated the major portion of his research career to the understanding of electronic current flow in semiconductors and semiconductor devices with an emphasis on effects pertinent to device miniaturization. He is currently the Swanlund Professor of Electrical and Computer Engineering, Professor of Physics, Adjunct Professor for Supercomputing Applications and a Research Professor in the Beckman Institute working on topics related to Molecular and Electronic Nanostructures. He has received numerous awards including the IEEE David Sarnoff Field Award for electronics in 1995.

Richard M. Martin is Professor of Physics at the University of Illinois, Urbana-Champaign. His area of research is theoretical condensed matter physics, with emphasis on the electronic structure of materials and quantum devices. The primary goals of his research are to predict the properties of real systems by simulating the many-body quantum system of electrons using density functional and quantum Monte Carlo methods and advanced computational methods. He received his Ph.D. in physics from the University of Chicago and has worked at Bell Laboratories and the Xerox Palo Alto Research Center. He is a fellow of the American Physical Society and the American Association for the Advancement of Science. 

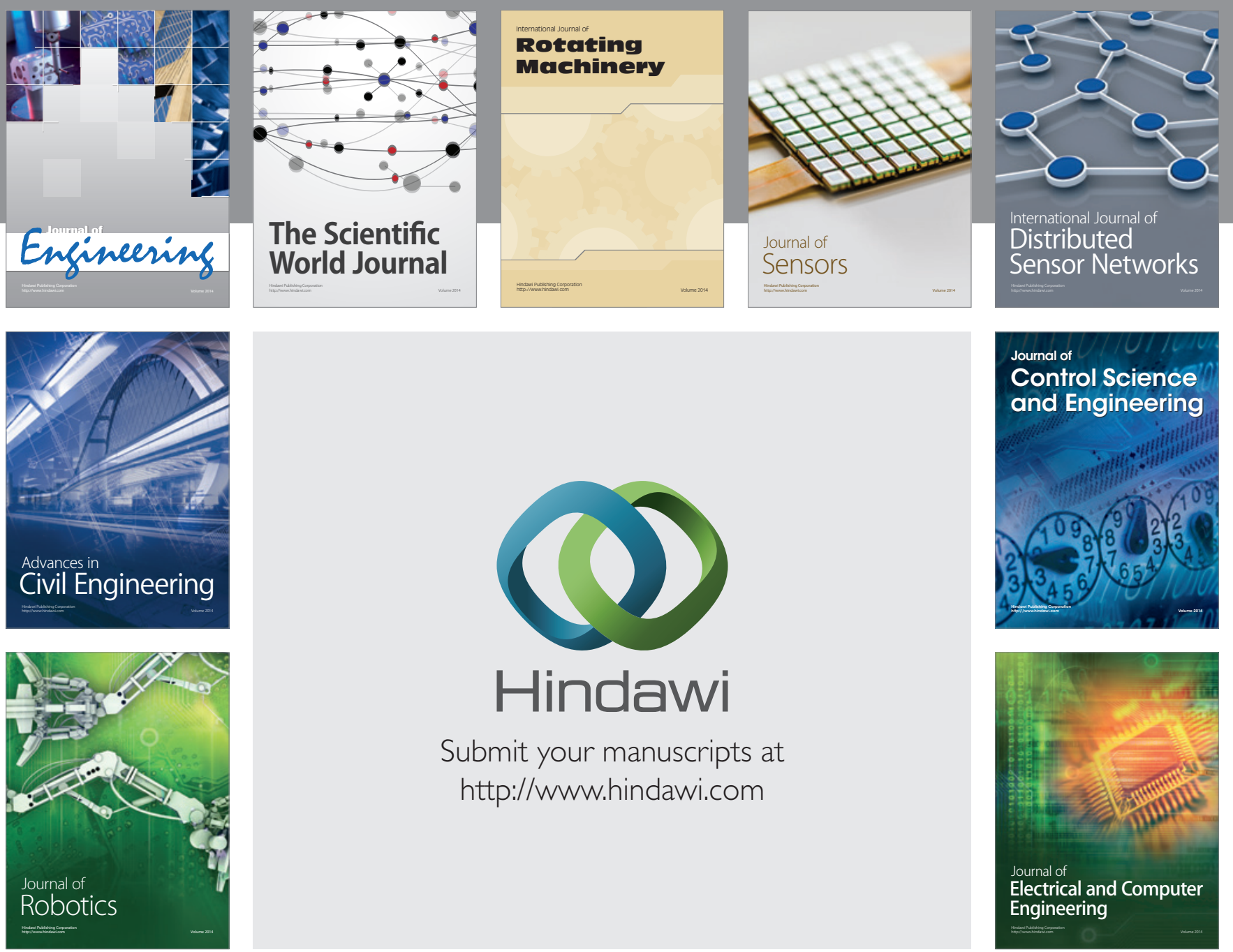

Submit your manuscripts at

http://www.hindawi.com
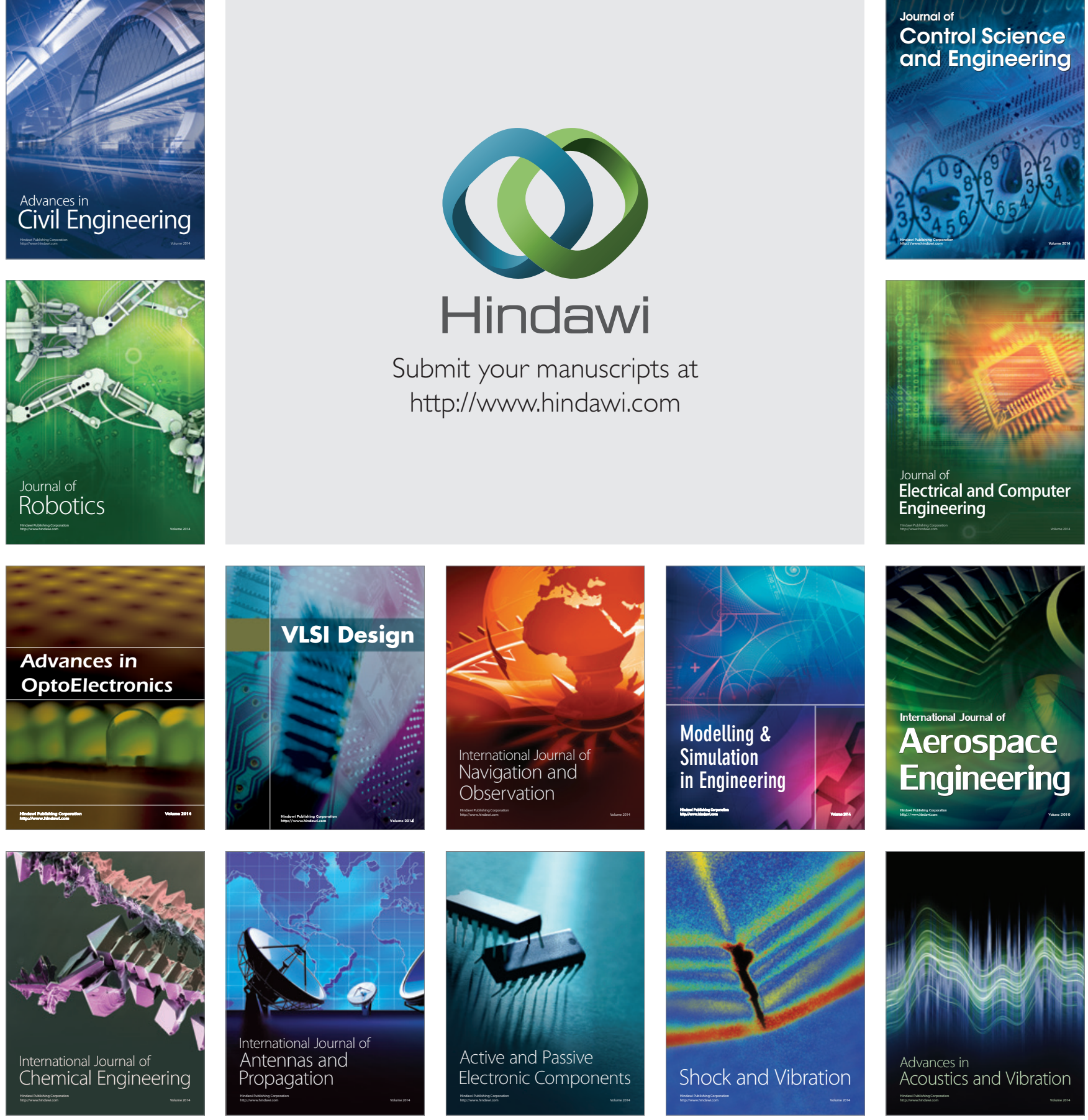